\title{
An Ultra-Compact Full-Band Waveguide Quadrature Hybrid Coupler
}

\author{
Juan Luis Cano ${ }^{\circledR}$, Gabriele Ceccato ${ }^{\circledR}$, Member, IEEE, Tomas Fernandez, Member, IEEE, \\ Angel Mediavilla ${ }^{\circledR}$, and Luca Perregrini ${ }^{\circledR}$, Fellow, IEEE
}

\begin{abstract}
This work proposes an easily scalable quadrature hybrid coupler (QHC) topology with two symmetric stepped apertures that originate a square coaxial-like mid-section. This new configuration enables an ultra-compact design, three times shorter than equivalent structures, having $40 \%$ of fractional bandwidth with $25 \mathrm{~dB}$ of matching/isolation and $1 \mathrm{~dB}$ of axial ratio (AR). The impedance transformers can be easily redesigned to meet other requirements related to electrical performance or even other desired coupling values. For verification purposes, a prototype at $\mathrm{Ku}$-band was manufactured and measured showing good agreement with simulation results.
\end{abstract}

Index Terms-Directional coupler, full bandwidth, hybrid coupler, waveguide.

\section{INTRODUCTION}

D IRECTIONAL couplers are one of the basic microwave circuits that require periodic attention since their capabilities of power division/combination or power sampling need to face more and more stringent requirements from new applications. Quadrature hybrid couplers (QHCs) are particularly important because they allow performing a quadrature correlation between the two input signals. Among the different technologies, waveguide is the best option for applications entailing low insertion loss and high-power handling characteristics such as antenna feed networks. Although waveguide hardware is bulky in nature, there is a growing interest in the design of ultra-compact structures with full-band performance that can be implemented in, for example, Butler matrices, multi-beam communication networks, or multi-pixel radio astronomy receivers [1], [2].

Riblet [3] coupler and its evolved version [4] are the classical option for compact devices in H-plane configuration. However, these structures rarely achieve a $25 \%$ of fractional bandwidth if reasonable electrical performance is pursued.

Manuscript received July 30, 2021; revised September 1, 2021; accepted September 26, 2021. This work was supported in part by the Spanish Ministry of Science, Innovation and Universities under Project TEC2017-83343-C4-1$\mathrm{R}$ and in part by European Fund for Economic and Regional Development (FEDER). (Corresponding author: Juan Luis Cano.)

Juan Luis Cano, Tomas Fernandez, and Angel Mediavilla are with the Department Ingeniería de Comunicaciones, Universidad de Cantabria, 39005 Santander, Spain (e-mail: juanluis.cano@unican.es; tomas.fernandez@ unican.es; angel.mediavilla@unican.es).

Gabriele Ceccato and Luca Perregrini are with the Department of Electrical, Computer and Biomedical Engineering, University of Pavia, 27100 Pavia, Italy (e-mail: gabriele.ceccato01@universitadipavia.it; luca.perregrini@unipv.it).

Color versions of one or more figures in this letter are available at https://doi.org/10.1109/LMWC.2021.3116103.

Digital Object Identifier 10.1109/LMWC.2021.3116103
In this context, it is possible to define good electrical performances as the input matching and isolation of, at least, $25 \mathrm{~dB}$ and the axial ratio (AR) better than $1 \mathrm{~dB}$. The AR is a figure of merit that gathers the amplitude and phase imbalances with respect to equal magnitude and $90^{\circ}$ out of phase signals [5]. Therefore, this is a very suitable parameter to characterize the performance of QHCs. When aiming at waveguide fullband coverage, $40 \%$ fractional bandwidth, and good electrical performances, E-plane structures offer more flexibility. Thus, couplers based on multi-hole [6], [7] or multi-window (branchline) [8], [9] couplings have demonstrated excellent responses, but at the cost of long coupling sections which are far from the compactness concept. A noticeable improvement in terms of compactness but maintaining good operational bandwidth has been achieved with the simple design in [10] as well as with [11] and the natural evolution [12]. In [13], the use of three apertures arranged in two different levels for the design of a $10 \mathrm{~dB}$ coupler achieves a $44 \%$ of bandwidth in a very compact configuration. However, it requires a number of matching fins and posts that increase the complexity at the design and manufacturing stages. These matching elements are unnecessary in the $10 \mathrm{~dB}$ coupler presented in [14], with a $42 \%$ bandwidth. Both Wang [13] and Xin [14] present only simulated results and do not aim at the design of a more challenging QHC, where the amplitude and phase imbalances are critical. In particular, [14] exhibits some mechanical limitations such as very thin metallic gaps between coupling apertures, that clearly limit the mechanization and frequency scaling, and a small waveguide height in the coupling section, which requires several steps in height to get the standard value, thus affecting its final compactness. In [15], the three apertures are positioned along the longitudinal axis obtaining a 41\%-bandwidth QHC but at the expense of increasing the total length. Finally, [16] presents an interesting configuration with two back-to-back septa originating a single multi-stepped coupling aperture that exhibits a high degree of compactness and good electrical characteristics but for a limited bandwidth of about $20 \%$.

To overcome the above drawbacks, this work proposes a new QHC architecture, close to [12], with two symmetric stepped apertures that originate a square coaxiallike mid-section (see Fig. 1). This configuration enables an ultra-compact design and broader bandwidth configuration. The proposed design does not use any additional impedance transformer at the output ports to reach the standard waveguide dimensions, and the internal structure can be easily redesigned to accomplish other requirements, concerning the electrical 

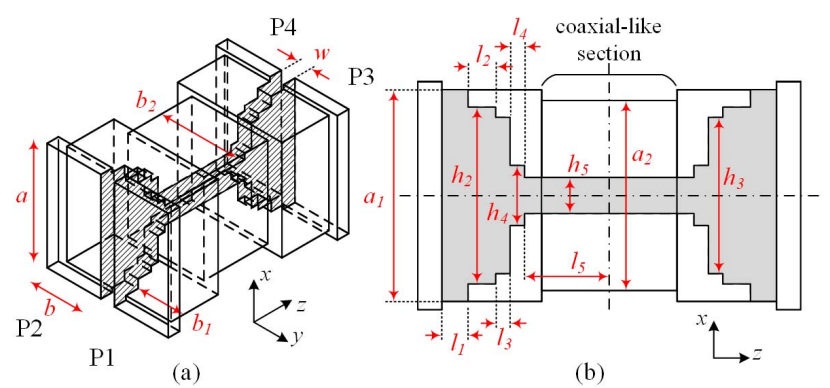

(b)

Fig. 1. (a) Three-dimensional and (b) side view of the proposed QHC with variables definition.

performance or the desired coupling level, with minimum modification of the total length. Moreover, the thickness of the internal coaxial-like section is thick enough to allow an easy mechanical migration toward higher frequencies.

\section{Compact Hybrid Coupler With CoAxial Section}

Unlike the coupler presented in [16], where the principle of operation is based on the septum polarizer mechanism and therefore, inherits its bandwidth limitations with a coupling value strongly dependent on the septum insertion phase, the hybrid proposed in this work overcomes such limitations. The coupling mechanism of the coupler in Fig. 1 is similar to structures with large coupling apertures [11]-[15], with a very stable phase balance over broad bandwidths and a coupling value much less sensitive to frequency variations, thus enabling full-band operation. Indeed, the phase error around $90^{\circ}$ can be mathematically demonstrated, following a similar procedure as in [5], to be negligible for the case of a good QHC, i.e., perfect symmetry and good matching and isolation with values better than $25 \mathrm{~dB}$. Thus, the design process is simplified to the minimization of the amplitude imbalance, which is carried out with the help of the steps in the ridge section. The frequency response is constrained, in the upper limit, by the appearance of high-order modes and, in the lower limit, by the acceptable coupler performance, which rapidly degrades when approaching the fundamental mode cut-off frequency.

\section{A. Field Analysis of the Coaxial-Like Section}

The quasi-squared central section shown in Fig. 1(b), with external dimensions $a_{2}$ and $b_{2}$, becomes a half-wave coaxiallike section, with a rectangular inner conductor of dimensions $h_{5}$ and $w$. As a consequence, the fundamental $\mathrm{TE}_{10}$ mode at the four ports of the coupler will be transformed into a quasi-TEM mode. In the central section, the electric field is not given by the only propagation of the TEM mode, but also few high order modes $\left(\mathrm{TE}_{10}\right.$-like, $\mathrm{TE}_{01}$-like, and the $\mathrm{TE}_{11}$-like modes) as shown in Fig. 2, resulting in a radial field directed from the metallic central section toward the outer metallic walls. Since the contribution of the high order modes is not negligible, the complete field in the mid-section will be the superimposition of the propagating modes, resulting in a quasi-TEM behavior, assuming a monomodal $\mathrm{TE}_{10}$ feeding

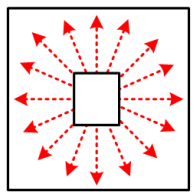

(a)

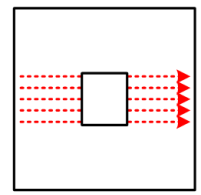

(b)

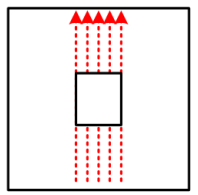

(c)

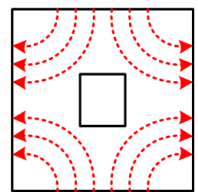

(d)
Fig. 2. Propagating modes in the coaxial section. (a) TEM mode. (b) $\mathrm{TE}_{10}$-like mode. (c) $\mathrm{TE}_{01}$-like mode. (d) $\mathrm{TE}_{11}$-like mode.

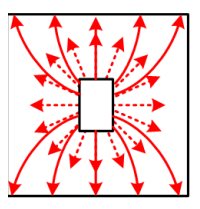

(a)

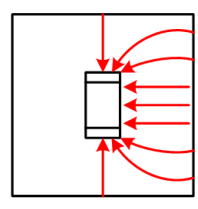

(b)

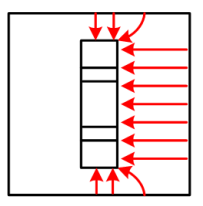

(c)

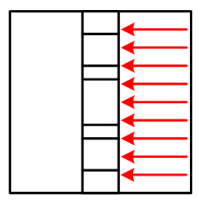

(d)
Fig. 3. Electric field propagating through the structure. (a) Quasi-TEM mode in section $h_{5}$ [see Fig. 1(b)]. (b) $E$-field in section $h_{4}$. (c) $E$-field in section $h_{3}$. (d) $E$-field in section $a_{1}$.

TABLE I

VARIABLES VALUES OF THE PROPOSED QHC. UNITS IN mm

\begin{tabular}{ccccccccc}
\hline \hline BW & $a_{1}$ & $a_{2}$ & $b_{1}$ & $b_{2}$ & $w$ & $l_{1}$ & $l_{2}$ & $l_{3}$ \\
\hline \multirow{3}{*}{$40 \%$} & 17.6 & 15.8 & 8 & 13.4 & 2.4 & 2.17 & 2.3 & 1.2 \\
\cline { 2 - 10 } & $l_{4}$ & $l_{5}$ & $h_{2}$ & $h_{3}$ & $h_{4}$ & $h_{5}$ & $L_{\text {tot }}$ & $A R$ \\
\cline { 2 - 9 } & 1.2 & 7.055 & 14.7 & 13 & 5 & 3 & 1.16 & 1 \\
\hline
\end{tabular}

$L_{t o t}$ is the total length of the coupling area divided by $\lambda_{0} . A R$ is the maximum axial ratio within the band in $\mathrm{dB}$. Ports have standard WR75 dimensions: $a=19.05 \mathrm{~mm}$ and $b=9.525 \mathrm{~mm}$.

at the input port. As the electric field propagates from one input port (i.e., $P_{1}$ ) to the output port (i.e., $P_{3}$ ), the complete sequence is shown in Fig. 3. Due to the symmetry of the structure, the same reasoning can be followed for all the other ports.

\section{B. Design of the Hybrid Coupler}

The proposed QHC is schematically depicted in Fig. 1. It is a structure with three planes of symmetry and consists of a central four-stepped ridge that separates the two rectangular waveguides building two large and symmetric coupling windows. The different steps in the ridge section act as an efficient impedance/mode transformer and they are obtained through a parametric study and optimization carried out using a fast mode-matching tool such as $\mu$ Wave Wizard from Mician [17]. The thickness of the ridge section, $w$, is constrained to a thick enough value, $\geq \lambda_{0} / 10$, being $\lambda_{0}$ the free-space wavelength at central frequency, so it enables an easy frequency scaling to over $100 \mathrm{GHz}$ by using traditional milling techniques. Finally, and due to the large value of parameter $b_{1}$, a key advantage of the proposed coupler is that only a single step is required to match the waveguide height in the coupling area to the standard rectangular waveguide usually required in the final application. This dramatically reduces the total QHC length regarding other more complex structures [13], [15]. The final parameters of the designed QHC having 40\% fractional bandwidth are provided in Table I, whereas the simulation results are presented together with the 


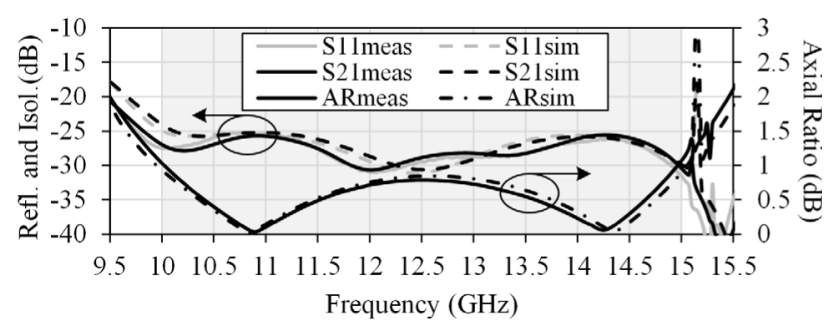

(a)

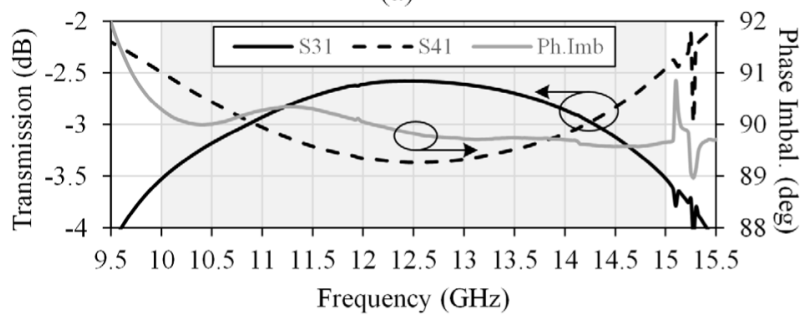

(b)

Fig. 4. (a) Measured and simulated reflection at input port $\left(S_{11}\right)$, isolation $\left(S_{21}\right)$, and AR results of the proposed QHC. (b) Measured transmissions $\left(S_{31}\right.$ and $\left.S_{41}\right)$ and phase imbalance. Design band in gray.

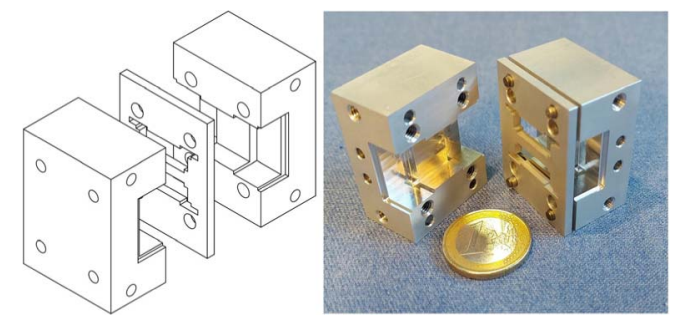

Fig. 5. Three-dimensional sketch and photograph of the designed QHC in $\mathrm{Ku}$-band.

measured results in Fig. 4 for a better comparison. These parameters can be easily reoptimized to accomplish other fractional bandwidths or coupling values resulting in similar $L_{\text {tot }}$ values, which gives an idea of the interesting flexibility of the proposed structure. The mechanical tolerance value is $\pm 20 \mu \mathrm{m}$, which is checked to not affect significantly the simulated results at $\mathrm{Ku}$-band after performing a yield analysis.

\section{Discussion ON COMPACTNESS}

The main contribution of the proposed QHC is the noticeable improvement in terms of compactness regarding alternative structures with similar electrical performances. In order to perform a fair comparison, which is shown in Table II, only $3 \mathrm{~dB}$ couplers aimed at around $-25 \mathrm{~dB}$ of reflection/isolation and $1 \mathrm{~dB}$ maximum of $\mathrm{AR}$ are compared. The total length is calculated between standard waveguide ports. Therefore, in the referenced works that do not provide information about the necessary impedance transformers, they have been calculated and included together with the declared coupling section length. For some topologies, and due to the lack of a suitable work to cite, a coupler has been designed based on the guidelines found in the available literature. Thus, a branch line coupler based on standard configurations [8], [9] is designed covering the $K u$-band (10-15 GHz) with the desired performance. This design requires a minimum
TABLE II

Comparison Between QHCs: Measured Performance

\begin{tabular}{ccccccc}
\hline \hline Ref. & $\begin{array}{c}S_{11} / I S \\
(\mathrm{~dB})\end{array}$ & $\begin{array}{c}\Delta A \\
(\mathrm{~dB})\end{array}$ & $\begin{array}{c}\Delta P h \\
(\mathrm{deg})\end{array}$ & $\begin{array}{c}A R \\
(\mathrm{~dB})\end{array}$ & $\begin{array}{c}\mathrm{BW} \\
(\%)\end{array}$ & $\begin{array}{c}L_{\text {tot }} \\
\left(\lambda_{0}\right)\end{array}$ \\
\hline$[8]^{*},[9]^{*}$ & -25 & \pm 0.5 & \pm 0.5 & 1 & 40 & 3.2 \\
\hline$[12]$ & -30 & \pm 0.5 & $\mathrm{~N} / \mathrm{A}$ & 1 & 26 & 1.58 \\
\hline$[14]^{*}$ & -25 & \pm 0.8 & \pm 0.4 & 1.5 & 40 & 4.25 \\
\hline$[15]$ & -25 & \pm 0.6 & $\mathrm{~N} / \mathrm{A}$ & $\mathrm{N} / \mathrm{A}$ & 41 & 4.22 \\
\hline$[16]$ & -25 & $\mathrm{~N} / \mathrm{A}$ & $\mathrm{N} / \mathrm{A}$ & 1 & 20 & 2.42 \\
\hline \hline This work & -25 & \pm 0.5 & \pm 0.5 & 1 & 40 & 1.16 \\
\hline \hline
\end{tabular}

* own design based on the referenced structure. $S_{I I}, I S, \triangle A, \triangle P h, A R, \mathrm{BW}$ and $L_{t o t}$ are the reflection, isolation, amplitude imbalance, phase imbalance, axial ratio, bandwidth and total length between standard waveguide ports with respect to free-space wavelength at central frequency, $\lambda_{0}$, respectively.

of eight coupling windows or branches. On the other side, a $3 \mathrm{~dB}$ coupler covering the whole $K u$-band has been designed following the compact configuration presented in [14] but it exhibits the limitations explained in the Introduction section. Finally, couplers in [12], [16] are very compact structures but with limited bandwidth. Therefore, the proposed QHC is three times shorter than any other equivalent structure.

\section{Manufacturing and Measurement Results}

The designed QHC covering the whole $K u$-band $(10-15 \mathrm{GHz})$ was mechanized in three aluminum parts as can be seen in Fig. 5: a central piece, which includes the two symmetrical coupling apertures along with the impedance transformers in ridge waveguide, and the two symmetric blocks that form the two parallel rectangular waveguides. Total dimensions are $40 \times 40 \times 30 \mathrm{~mm}^{3}$ and the output ports are standard WR75.

In order to be able to measure the performance of the coupler with standard waveguide hardware, additional pieces are required. These pieces include suitable waveguide bends so the output ports are conveniently spaced to allow the connection of two standard WR75 flanges in parallel. Then the device is measured using a vector network analyzer (VNA), after a TRL calibration at the WR75 flanges plane, connected to the desired two ports and waveguide loads in the remaining ports. Some measured results are presented in Fig. 4. As can be seen, the input matching and isolation values are below $-25 \mathrm{~dB}$ in the whole frequency range of interest whereas the $\mathrm{AR}$ is better than $1 \mathrm{~dB}$ as expected from simulations. The spikes above $15 \mathrm{GHz}$ are due to the appearance of high-order modes. The insertion loss is calculated to be less than $0.03 \mathrm{~dB}$, after eliminating the $-3 \mathrm{~dB}$ split ratio and the phase imbalance is limited to $\pm 0.5^{\circ}$ as shown in Fig. 4(b).

\section{Conclusion}

A new coupling architecture that results in an extremely compact quadrature E-plane hybrid circuit has been experimentally demonstrated in $\mathrm{Ku}$-band. The proposed QHC represents a clear step forward in the current state of the art in terms of compactness and bandwidth. The existence of a thick internal coaxial-like section allows full waveguide band operation and easy mechanical scaling beyond $100 \mathrm{GHz}$, while maintaining good electrical performance and easy mechanization. 


\section{REFERENCES}

[1] J. Remez and R. Carmon, "Compact designs of waveguide Butler matrices," IEEE Antennas Wireless Propag. Lett., vol. 5, pp. 27-31, 2006.

[2] E. Villa et al., "Polarimetric receiver in the forty gigahertz band: New instrument for the Q-U-I joint Tenerife experiment," Exp. Astron., vol. 45, pp. 127-146, Feb. 2018.

[3] H. Riblet, "The short-slot hybrid junction," Proc. IRE, vol. 40, no. 2, pp. 180-184, Feb. 1952.

[4] L. T. Hildebrand, "Results for a simple compact narrow-wall directional coupler," IEEE Microw. Guided Wave Lett., vol. 10, no. 6, pp. 231-232, Jun. 2000.

[5] J. L. Cano and A. Mediavilla, "On the accurate full characterization of septum polarizers through simple amplitude measurements in back-toback configuration," IEEE Trans. Microw. Theory Techn., vol. 69, no. 1, pp. $179-188$, Jan. 2021.

[6] S. Wen, Q. Wang, Taowu, and Y. Tan, "Design of a compact $3 \mathrm{~dB}$ Ka-band directional coupler," in Proc. Int. Workshop Microw., Millim. Wave Circuits Syst. Technol., Chengdu, China, Apr. 2012, pp. 1-4.

[7] M. M. M. Ali, S. I. Shams, and A.-R. Sebak, "Low loss and ultra flat rectangular waveguide harmonic coupler," IEEE Access, vol. 6, pp. 38736-38744, 2018.

[8] S. Srikanth and A. Kerr, "Waveguide quadrature hybrids for ALMA receivers," Nat. Radio Astron. Observatory, Charlottesville, VA, USA, Tech. Rep. ALMA Memo \#343, 2001.
[9] H. Rashid, V. Desmaris, V. Belitsky, M. Ruf, T. Bednorz, and A. Henkel, "Design of wideband waveguide hybrid with ultra-low amplitude imbalance," IEEE Trans. THz Sci. Technol., vol. 6, no. 1, pp. 83-90, Jan. 2016.

[10] T. Kawai, M. Kishihara, Y. Kokubo, and T. Ohta, "Cavity-type directional couplers with simple structure," in IEEE MTT-S Int. Microw. Symp. Dig., Denver, CO, USA, Jun. 1997, pp. 413-416.

[11] E. Hadge, "Compact top-wall hybrid junction," Trans. IRE Prof. Group Microw. Theory Techn., vol. 1, no. 1, pp. 29-30, Mar. 1953.

[12] R. Beyer and U. Rosenberg, "Compact top-wall hybrid/coupler design for extreme broad bandwidth applications," in IEEE MTT-S Int. Microw. Symp. Dig., Long Beach, CA, USA, Jun. 2005, pp. 1227-1230.

[13] C. L. Wang, "Compact $10 \mathrm{~dB}$ broadband directional coupler," in Proc. IEEE Int. Conf. Appl. Superconductivity Electromagn. Devices, Beijing, China, Oct. 2013, pp. 282-284.

[14] H. Xin, S. Li, and Y. Wang, "A terahertz-band E-plane waveguide directional coupler with broad bandwidth," in Proc. 16th Int. Conf. Electron. Packag. Technol., Changsha, China, Aug. 2015, pp. 1419-1421.

[15] Y. Zhang, Q. Wang, and H. Xin, "A compact $3 \mathrm{~dB}$ E-plane waveguide directional coupler with full bandwidth," IEEE Microw. Wireless Comp. Lett., vol. 24, no. 4, pp. 227-229, Apr. 2014.

[16] N. J. G. Fonseca and J.-C. Angevain, "Waveguide hybrid septum coupler," IEEE Trans. Microw. Theory Techn., vol. 69, no. 6, pp. 3030-3036, Jun. 2021.

[17] MICIAN GmbH. Mician WaveWizard. Accessed: Jul. 30, 2021. [Online]. Available: https://www.mician.com/ 